\title{
An efficient technique to solve combined economic and emission dispatch problem using modified Ant colony optimization
}

\author{
R GOPALAKRISHNAN* and A KRISHNAN \\ Department of Electrical and Electronics Engineering, K S Rangasamy College \\ of Technology, Tiruchengode 637 215, India \\ e-mail: gopsengr@gmail.com; amasikrishnan@hotmail.com
}

MS received 31 October 2011; accepted 30 May 2013

\begin{abstract}
Economic load dispatch is one of the vital purposes in electrical power system operation, management and planning. Economic dispatch problem is one of the most important problems in electric power system operation. In large scale system, the problem is more complex and difficult to find out optimal solution because it is nonlinear function and it contains number of local optimal. Combined economic emission dispatch (CEED) problem is to schedule the committed generating units outputs to meet the required load demand at minimum operating cost with minimum emission simultaneously. The main aim of economic load dispatch is to reduce the total production cost of the generating system and at the same time the necessary equality and inequality constraints should also be fulfilled. This leads to the development of CEED techniques. There are various techniques proposed by several researchers to solve CEED problem based on optimization techniques. But still some problems such as slower convergence and higher computational complexity exist in using the optimization techniques such as GA for solving CEED problem. This paper proposes an efficient and reliable technique for combined fuel cost economic optimization and emission dispatch using the Modified Ant Colony Optimization algorithm (MACO) to produce better optimal solution. The simulation results reveal the significant performance of the proposed MACO approach.
\end{abstract}

Keywords. Combined economic emission dispatch (CEED); optimization algorithms; power demand; Ant colony optimization; modified Ant colony optimization (MACO).

\section{Introduction}

The advantages of minimum production cost, maximum reliability and better operating conditions are obtained through interconnected electric utility system. Economic scheduling is the

*For correspondence 
on-line economic dispatch (Chowdhury Badrul \& Rahman 1990; Wong \& Yuryevich 1998), in which it is needed to distribute the load among the generating units which are actually paralleled with the system, in such a way as to reduce the total cost of generating. It is assumed that some flexibility exists in adjusting the power delivered by each generator (Alrashidi \& El-Hawary 2008; Wong \& Yuryevich 1998).

The major issues of the electrical energy supply system are concerned with the efficiency on the generator, transmission, and distribution system (Singh \& Dhillon 2008). Solving the above mentioned issues on reducing the operational cost of fuel consumption has become the objective function and other requirements as the constraints.

Owing to strict governmental regulations on environment protection, the traditional techniques at absolute minimum fuel cost cannot be the only foundation for dispatching electric power (Abido 2003).

Recently, energy resources such as fossil fuels were used to generate mechanical power supplied to the rotor shaft of generating units (Lowery 1966; Ayoub \& Patton 1971). The production of electricity from fossil fuel discharges various pollutants like sulphur oxide (SO), and oxides of nitrogen $\left(\mathrm{NO}_{\mathrm{X}}\right)$ into the atmosphere (Song et al 1995, 1997). These pollutants can also cause a greenhouse effect which can result in global warming on the surface of the earth with other side effects. These gaseous pollutants result in harmful effects on human beings and also on plants and animals.

There are vital necessity for sufficient and secure electricity at the cheapest possible price with minimum levels of pollution. It is important for electric utilities to minimize pollution level by minimizing emission of $\mathrm{CO}, \mathrm{CO}_{2}, \mathrm{SO}_{2}$ and $\mathrm{NO}_{\mathrm{x}}$. There are various techniques available in the literature for minimizing emissions (King et al 1995; Chaturvedi et al 2008). But, the major drawbacks of the existing techniques are switching to low sulphur content in coal, installing postcombustion cleaning system, allocation of generation to each generator unit with the objective to minimum emission dispatch (Talaq et al 1994).

In the case of economic load dispatch, the generations are not fixed, they are allowed to take values of economic load dispatch problem pertaining to the optimum generation scheduling of available generating units in a power system to minimize the cost of generation subject to system constraints (Wong \& Yuryevich 1998). Recently, optimization algorithms (Ratniyomchai et al 2010) are found to be very effective in providing better optimized solution to the emission and economic dispatch problem. Genetic Algorithm (GA) is one of the significant optimization algorithms used to solve CEED problem (Liladhur et al 2004; Ugur Guvenc 2010). But, GA suffers from slower convergence rate and higher computational cost which would affect the overall performance. More recently, Ant colony algorithms which were inspired by the observation of real Ant colonies are observed to provide better optimized results than GA.

This paper uses modified Ant colony optimization (MACO) approach to reduce the total system operating cost and emission levels. To anticipate the pollutant problem, the proposed MACO algorithm contains two objective functions, i.e., economic objective function (fuel cost and transmission losses) and emission objective function.

\section{Literature survey}

The Harmony Search (HS) technique was initially conceptualized with the help of the musical improvization method of searching for a perfect state of harmony. The HS technique utilizes a random search that is based on random selection, memory consideration and pitch adjusting. Dos Santos Coelho et al (2010) provide a modified HS technique incorporated with differential 
evolution and chaotic sequences to resolve the economic load dispatch difficulty of thermal generators with the valve-point effect. The provided modified HS algorithm was validated in a power economic problem that consists of 10 generating units with valve-point effects and multiple fuels for a load demand of $2500 \mathrm{MW}$. Experimental results indicate that the modified HS algorithm resulted in satisfied outcome when compared with outcome of other optimization techniques.

Effective and best economic operations of electric power generation systems have always been considered important in the electric power industry. This involves assigning of the total load between the available generating units such that the total cost of operation is kept at a minimum. Recently, this problem has taken an appropriate twist as the public concern for the environmental matters has increased and thus, economic dispatch now includes the dispatch of systems to reduce pollutants while achieving minimum cost. Lakshmi Devi \& Vamsi Krishna (2008) propose a lambda-based technique for solving the CEED problem using Genetic Algorithm (GA) and Particle Swarm Optimization (PSO) techniques considering the power limits of the generator. The proposed approach determines the global or near-global optimal solution for the CEED problem. The main aim of CEED is to reduce both the operating fuel cost and emission level simultaneously while maintaining load demand and operational constraints. This multi-objective CEED problem is converted into a single objective function using a modified price penalty factor technique. This approach is tested with three generating units. The PSO technique has shown to offer accurate and feasible solutions within acceptable computation time.

To overcome the economic dispatch difficulty in power system, Peng Chen et al (2009) proposed the floating point representation to the Genetic Particle Swarm Optimization (GPSO). GPSO was a derivative of the Standard Particle Swarm Optimization (SPSO) and combined with the genetic reproduction techniques called crossover and mutation. An altered heuristic crossover was developed that was obtained from the differential evolution and genetic algorithm together with the technique of GPSO. The presented technique was implemented to four well-known benchmark functions, and typical constraint sets were provided according to the evaluation outcome. Additionally, MGPSO was utilized to a practical system, and by assessment with the other PSO techniques, MGPSO has resulted in a better solution.

\section{Methodology}

\subsection{Economic dispatch}

The economic dispatch problem is to determine the optimal mixture of power generation in a manner that the entire production cost of the entire system is reduced while satisfying the total power demand and few key power system factors. The fuel cost for all the power generation unit is defined initially. Therefore, the total production cost function of economic dispatch problem is defined as the total sum of the fuel costs of all the generating plant units as mentioned below:

$$
F_{T}=\sum_{i=1}^{N_{G}}\left\{a_{i} P_{i}^{2}+b_{i} P_{i}+c_{i}\left|d_{i} \operatorname{sine}_{i}\left(P_{i}^{\min }-P_{i}\right)\right|\right\},
$$

where

$N_{G}$ is the total number of generating units

$F_{T}$ is the total production cost

$P_{i}$ is the power output of generating unit $i$ 
$P_{i}^{\min }$ is the minimum output of generating unit $i$

$a_{i}, b_{i}, c_{i}, d_{i}, e_{i}$ are fuel cost coefficients of unit $i$

This equation helps in determining the total production cost of the generating plant.

\subsection{Emission dispatch}

The solution of economic dispatch problem will provide the quantity of active power to be produced by various units at the minimum production cost for a certain power requirement. On the other hand, the total quantity of pollutant emission is not considered in conventional economic dispatch problem. The quantity of pollutant emission resulting from a fossil-fired thermal generating unit is based on the amount of power generated by every unit. For reducing the complexity, the total emission produced can be modelled as a direct sum of a quadratic function and an exponential term of the active power output of the generating units. The pollutant emission dispatch problem can be described as the optimization of total amount of pollutant emission given as below:

$$
E_{T}=\sum_{i=1}^{N_{G}}\left\{a_{i} P_{i}^{2}+\beta P_{i}+\gamma_{i}+\xi_{i} e^{\tau_{i} P_{i}}\right\},
$$

where

$N_{G}$ is the total number of generating units

$E_{T}$ is the total pollutant emission

$P_{i}$ is the power output of generating unit $i$

$a_{i}, \beta_{i}, \gamma_{i}, \xi_{i}, \tau_{i}$ are emission coefficients of unit $i$.

\subsection{Combined economic and emission dispatch}

The economic dispatch and emission dispatch are two various problems as discussed previously. Emission dispatch can be included in conventional economic load dispatch problems by merging an emission constraint with the economic load dispatch problem (Dhillon et al 1993; Alrashidi \& El-Hawary 2008). In this study, the two objectives can be converted into a single objective function by introducing a price penalty factor as defined follows.

$$
h=\frac{F_{T}\left(P_{i}^{\max }\right) / P_{i}^{\max }}{E_{T}\left(P_{j}^{\max }\right) / P_{j}^{\max }},
$$

where

$h$ is the price penalty factor

$i$ is the highest fuel-cost unit

$j$ is the highest pollutant-emission unit.

The combined objective function of the economic and emission dispatches is represented by the following equation:

$$
\Phi_{T}=w_{\text {eco }} F_{T}+w_{\text {emi }} h E_{T},
$$

where

$\Phi_{T}$ is the combined objective function

$w_{\text {eco }}, w_{\text {emi }}$ are weighting factors.

The two weighting factors can be provided in various ways. The case of $w_{e c o},=1.0$ and $w_{\text {emi }}=0.0$ used to obtain the classical economic dispatch problem and the pure emission 
dispatch results when $w_{\text {eco }},=0.0$ and $w_{\text {emi }}=1.0$. To obtain the combined economic and emission dispatch problem, both weighting factors should be equal, for example $w_{\text {eco }},=0.5$ and $w_{\text {emi }}=0.5$.

\subsection{Problem constraints}

Usually, two constraints such as equality and inequality constraints should be considered. For the problem defined in this study, a power balance equation (5) is set as an equality constraint and the limits of power generation output (6) are set as inequality constraints.

$$
\begin{gathered}
P_{D}+P_{L O S S}-\sum_{i=1}^{N_{G}} P_{i}=0 \\
P_{i}^{\min } \leq P_{i} \leq P_{i}^{\max }, i=1,2, \ldots, N_{G},
\end{gathered}
$$

where

$P_{D}$ is the total power demand of the plant

$P_{L O S S}$ is the total power losses of the plant

$P_{i}^{\min }$ is the minimum output of generating unit $i$

$P_{i}^{\max }$ is the maximum output of generating unit $i$.

With this defined problem and objective function, an appropriate optimization technique is used to obtain the required objective. This paper uses Modified Ant Colony optimization technique has various advantages over the existing system which uses conventional optimization techniques to solve the CEED problem.

\subsection{Ant colony optimization}

Ant Colony Optimization (ACO) (Dorigo et al 2006; AL-salami Nada \& Saad Ghaleb Yaseen 2008 ) is a metaheuristic algorithm for providing solutions to combinational optimization problems through the principles of communication on the paths to find food sources by depositing pheromone in the paths. Pheromone trails can lead other ants to food sources.

ACO has been motivated by the behaviour of real ants which were capable of choosing the shortest path between their nest and food resources, in the presence of alternate paths between the food and the nest (Sandalidis et al 2004; Fernando \& Teresa 2008). Ants leave a pheromone substance along the path from the nest to food. When the ants arrive at a decision point, they make a probabilistic choice based on the intensity of pheromone substance. The probability of selecting the same path is higher due to increase intensity of pheromone substance. The new pheromone will be released on the chosen path. This behaviour has an autocatalytic effect as the very fact of selecting a path will increase the amount of pheromone substance on the corresponding path which makes it more attractive for future ants to follow. Shortly, all ants will choose the shortest path (Hui Fan et al 2004).

3.5a ACO Method: ACO is the random search technique. Usually, an ACO approach can be applied to any combination problem if the following factors are defined:

- Appropriate problem representation: The problem can be illustrated as a graph with a group of nodes and edges between nodes.

- Heuristic desirability (P) of edges: An appropriate heuristic measure of the 'goodness' of paths from one node to every other associated node in the graph. 
- Construction of feasible solutions: A mechanism must be in place whereby possible solutions are efficiently created.

- Pheromone updating rule: An appropriate technique of updating the pheromone levels on edges is needed with an equivalent trail update rule (Song et al 1997).

3.5b Basic Ant colony algorithm operation: The fundamental principle of the technique is to have a population of artificial Ants that cyclically construct a solution to a combinational optimization. The ants move along every branch from one node to another node and so build paths representing solutions. Starting in an initial node, every Ant chooses the next node in its path according to trail update and state transition rule (Wong \& Yuryevich 1998).

3.5c Trail Update: Let $\tau i j(t)$ be the intensity of the trail on edge $(i, j)$ at time $t$. After $n$ iterations of the algorithm the trail intensity becomes

$$
\tau_{i j}(\tau+n)=\rho \cdot \tau_{i j}(t)+\Delta \tau_{i j}(t, t+n),
$$

where $\rho$ is a coefficient such that $(1-\rho)$ representations of the evaporation of trail between time $t$ and time $t+n$ and

$$
\tau_{i j}(t, t+n)=\sum_{k=1}^{m} \Delta \tau_{i j}^{k}(t, t+n),
$$

where $\Delta \tau_{i j}^{k}$ is the quantity per unit length of trail substance laid on edge $(i, j)$ between time $t$ and $t+n$ by Ant $k$. Pheromone values are updated on edge $(i, j)$ every time an ant moves from node $i$ to node $j$. The amount of new pheromone added to the edge is equal to

$$
\begin{aligned}
& \Delta \tau_{i j}^{k}(t, t+1) \\
& \quad=\left\{\begin{array}{c}
Q \text { if the } k^{\text {th }} \text { ant goes from node } i \text { to } j b / t \text { time } t \text { and } t+1 \\
0 \text { otherwise, }
\end{array}\right.
\end{aligned}
$$

where $Q$ is some constant quantity of pheromone (Wong \& Yuryevich 1998).

3.5d Transition probability: The transition probability from node $i$ to $j$ for $k^{\text {th }}$ ant is

$$
p_{i j}^{k}(t)=\frac{\left[\tau_{i j}^{k}\right]^{\alpha}\left[\eta_{i j}\right]^{\beta}}{\sum\left[\tau_{i u}^{k}(t)\right]^{\alpha}\left[\eta_{i u}\right]^{\beta}} .
$$

where allowed means that ( $j$ is not in table of ant $k$ ) and $\eta_{i j}$ is the visibility of node $j$ from node $i$ and it varies according to the processed application.

The transition probability is a trade-off between visibility (which says that process should be chosen with high probability, thus implementing a greedy constructive heuristic) and trail intensity at time $t$ (that says that if on edge $(i, j)$ there has been much traffic then it is highly desirable, thus implementing an autocatalytic process). The value $\alpha$ and $\beta$ are parameters that control the effect of trail and visibility on the transition, respectively. By manipulating the value of $\alpha$ and $\beta$ one can transform the transition probability from greedy heuristic that values visibility over trail $(\beta \gg \alpha)$ values are approximately in the same range, then best results have been found. 
The quantities of $\alpha$ and $\beta$ are parameters of the algorithm. The quantity $\tau_{i j}^{k}(t)$, is the pheromone concentration. The pheromone level of each pair of sections is updated at the end of each trail when the Ant has generated a valid dispatch of generated powers.

3.5e Modified Ant colony algorithm: The Ant algorithm is modified to provide better and significant results. The Ant algorithm system is characterized by a number of controlling parameters such as the sensitivity to the pheromone concentration $(\alpha)$, the sensitivity to cost of the path $(\beta)$, the number of iterations of the algorithm and the number of Ants used per iteration.

For the parameters $\alpha$ and $\beta$ in the transition probability rule, best results are found when both values are approximately in the same range, but there was a problem when the range of the pheromone value and visibility values are far from each other. So, in this case, the parameters $\alpha$ and $\beta$ are selected in such a way that the range of pheromone values and visibility values are close to each other. This approximation can be found in the $\exp (x)$ and $10 \log (x)$ functions, where $x$ is any value.

So the first modified transition probability can be written as:

$$
p_{i j}^{k}(t)=\left\{\begin{array}{c}
\frac{\exp \left(\tau_{i j}^{k}(t)\right) * \exp \left(\eta_{i j}(t)\right)}{\sum \exp \left(\tau_{i u}(t)\right) * \exp \left(\eta_{i u}(t)\right)} u \in \text { allowed } \\
0 \text { otherwise }
\end{array} .\right.
$$

The exponential function is characteristic by its large rate increase in values. That characteristic can be used to obtain the best result rapidly. The second modified transition probability can be written as:

$$
p_{i j}^{k}(t)=\left\{\begin{array}{c}
\frac{\log _{10}\left(1+\tau_{i j}(t)\right) * \log _{10}\left(1+\eta_{i j}(t)\right)}{\sum \log _{10}\left(1+\tau_{i u}(t)\right) * \log _{10}\left(1+\eta_{i u}(t)\right)} u \in \text { allowed } \\
0 \text { otherwise }
\end{array} .\right.
$$

The logarithmic function is characteristic by converting a large range of values to a small range of values, so the two parameters $\alpha$ and $\beta$ are not required. Here, using any one of the above two equations will reduce the control parameters by eliminating the parameters $\alpha$ and $\beta$ and in the same time reducing the number of Ant algorithm iterations which result in increasing the system performance (Wong \& Yuryevich 1998).

Finally, the optimized parameters will help in achieving the solution for CEED problem. The evaluation of the proposed technique for CEED is presented in the simulation results.

\section{Simulation results}

The proposed method for CEED problem is evaluated using the six-generator system. Fuel cost coefficients and generation limits for each generating unit of the test system are given in table 1 and the emission coefficient is given in table 2 . The proposed technique is tested by varying the power demand such as $400 \mathrm{MW}, 500 \mathrm{MW}, 600 \mathrm{MW}, 700 \mathrm{MW}, 800 \mathrm{MW}, 900 \mathrm{MW}$ and $1000 \mathrm{MW}$. The resulted CEED solution for the considered six-generator system is presented in table 3 . From the table, it can be observed that the cost required for generating the required power increases as the power demand rises. Also, the emission output rises as the power demand rises.

The fuel cost required for particular power demand and the resulted emission output by using the proposed technique can be found in table 3. The proposed MACO technique is evaluated 
Table 1. Fuel cost coefficients for six-generator system.

\begin{tabular}{lccccccc}
\hline Generator & $\mathrm{a}$ & $\mathrm{b}$ & $\mathrm{c}$ & $\mathrm{d}$ & $\mathrm{e}$ & $P_{\min }$ & $P_{\max }$ \\
\hline 1 & 100 & 200 & 12 & 10 & 8.45 & 10 & 180 \\
2 & 110 & 190 & 15 & 20 & 11.45 & 15 & 200 \\
3 & 115 & 210 & 10 & 10 & 14.87 & 18 & 220 \\
4 & 90 & 195 & 10 & 15 & 17.54 & 25 & 250 \\
5 & 105 & 180 & 20 & 10 & 16.69 & 35 & 350 \\
6 & 120 & 170 & 15 & 20 & 20.21 & 50 & 400 \\
\hline
\end{tabular}

Table 2. Emission coefficients for six-generator system.

\begin{tabular}{lccccc}
\hline Generator & $\alpha$ & $\beta$ & $\gamma$ & $\xi$ & $\mathrm{T}$ \\
\hline 1 & 5.743 & -5.076 & 3.870 & $2 \times 10^{-4}$ & 4.678 \\
2 & 5.194 & -5.987 & 4.765 & $3 \times 10^{-4}$ & 3.034 \\
3 & 6.542 & -4.054 & 4.353 & $3 \times 10^{-4}$ & 7.361 \\
4 & 5.764 & -5.875 & 5.769 & $4 \times 10^{-4}$ & 5.908 \\
5 & 7.109 & -6.045 & 3.812 & $1 \times 10^{-6}$ & 6.210 \\
6 & 5.431 & -5.342 & 4.873 & $2 \times 10^{-4}$ & 4.890 \\
\hline
\end{tabular}

Table 3. CEED solution of six-generator system.

\begin{tabular}{lrrrrrrr}
\hline & \multicolumn{7}{c}{ Power Demand (MW) } \\
\cline { 2 - 8 } & \multicolumn{1}{c}{400} & \multicolumn{1}{c}{500} & \multicolumn{1}{c}{600} & \multicolumn{1}{c}{700} & \multicolumn{1}{c}{800} & \multicolumn{1}{c}{900} & \multicolumn{1}{c}{1000} \\
\hline Unit 1 & 15.01 & 19.21 & 24.26 & 39.54 & 45.19 & 65.21 & 69.21 \\
Unit 2 & 19.21 & 28.35 & 35.75 & 65.31 & 74.18 & 91.56 & 103.51 \\
Unit 3 & 51.09 & 65.05 & 71.51 & 96.09 & 102.43 & 128.45 & 135.43 \\
Unit 4 & 89.11 & 103.96 & 135.34 & 149.62 & 161.51 & 175.97 & 187.41 \\
Unit 5 & 98.07 & 124.33 & 147.23 & 161.32 & 194.57 & 208.37 & 228.85 \\
Unit 6 & 127.51 & 159.10 & 185.91 & 188.12 & 222.12 & 230.44 & 275.59 \\
Fuel Cost $(\$ / h)$ & 28564.87 & 34674.12 & 37912.65 & 42632.98 & 47923.64 & 52543.76 & 59721.54 \\
Emission Output $(\mathrm{Kg} / h)$ & 253.34 & 301.56 & 405.54 & 521.56 & 645.56 & 721.87 & 879.34 \\
\hline
\end{tabular}

Table 4. Required fuel cost $(\$ / h)$ for using different techniques.

\begin{tabular}{lccccccc}
\hline Optimization & \multicolumn{7}{c}{ Power Demand (MW) } \\
\cline { 2 - 8 } Technique & 400 & 500 & 600 & 700 & 800 & 900 & 1000 \\
\hline PSO & 28567.11 & 34676.45 & 37915.11 & 42635.23 & 47925.23 & 52545.35 & 59724.12 \\
GA & 28565.24 & 34675.04 & 37913.54 & 42634.23 & 47924.45 & 52544.81 & 59724.32 \\
NRGA & 28564.87 & 34674.12 & 37912.65 & 42632.98 & 47923.64 & 52543.76 & 59721.54 \\
MACO & 28561.12 & 34671.34 & 37910.51 & 42630.21 & 47921.51 & 52541.12 & 59719.26 \\
\hline
\end{tabular}


Table 5. Emission output $(\mathrm{kg} / \mathrm{h})$ for using different techniques.

\begin{tabular}{lccccccr}
\hline Optimization & \multicolumn{7}{c}{ Power Demand (MW) } \\
\cline { 2 - 8 } Technique & 400 & 500 & 600 & 700 & 800 & 900 & 1000 \\
\hline PSO & 254.89 & 303.98 & 406.78 & 524.98 & 647.91 & 723.17 & 882.89 \\
GA & 254.45 & 303.12 & 406.23 & 522.19 & 647.62 & 722.45 & 882.32 \\
NRGA & 253.34 & 301.56 & 405.54 & 521.56 & 645.56 & 721.87 & 879.34 \\
MACO & 251.12 & 299.14 & 403.11 & 519.02 & 646.07 & 718.13 & 876.14 \\
\hline
\end{tabular}

Table 6. Number of iterations required by various methods.

\begin{tabular}{lrrcc}
\hline Power Demand (MW) & PSO & GA & NRGA & MACO \\
\hline 400 & 120 & 91 & 65 & 42 \\
500 & 126 & 106 & 71 & 53 \\
600 & 135 & 110 & 69 & 48 \\
700 & 141 & 121 & 74 & 51 \\
800 & 136 & 119 & 61 & 41 \\
900 & 135 & 125 & 71 & 43 \\
1000 & 151 & 137 & 83 & 53 \\
\hline
\end{tabular}

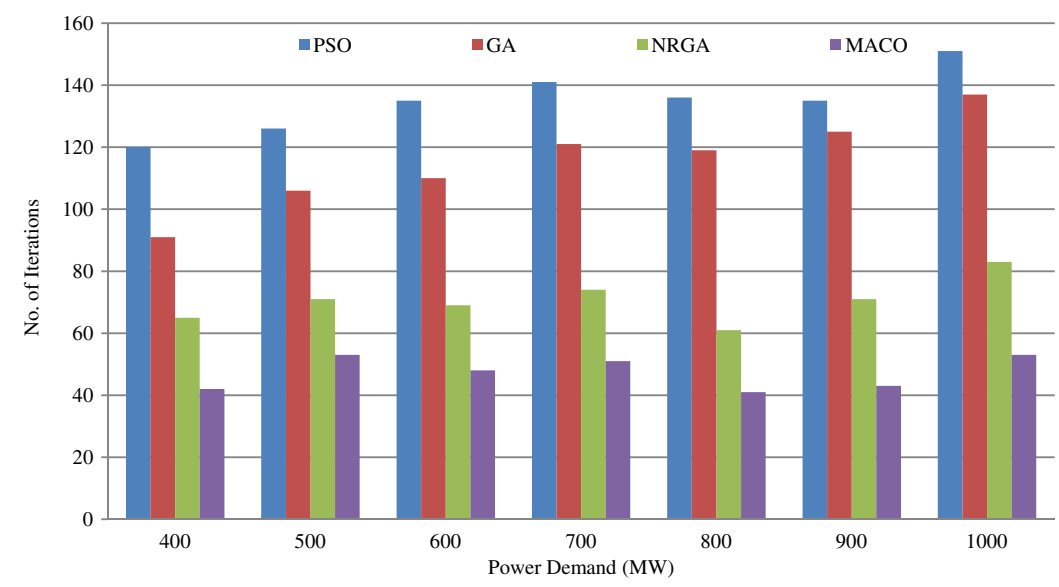

Figure 1. Comparison of the proposed technique by means of number of required iterations.

Table 7. Required CPU time (ms) by various methods.

\begin{tabular}{lcccc}
\hline Power demand (MW) & PSO & GA & NRGA & MACO \\
\hline 400 & 246 & 198 & 151 & 98 \\
500 & 251 & 202 & 163 & 110 \\
600 & 262 & 210 & 149 & 121 \\
700 & 234 & 187 & 156 & 128 \\
800 & 278 & 201 & 161 & 135 \\
900 & 281 & 199 & 164 & 144 \\
1000 & 298 & 215 & 172 & 153 \\
\hline
\end{tabular}




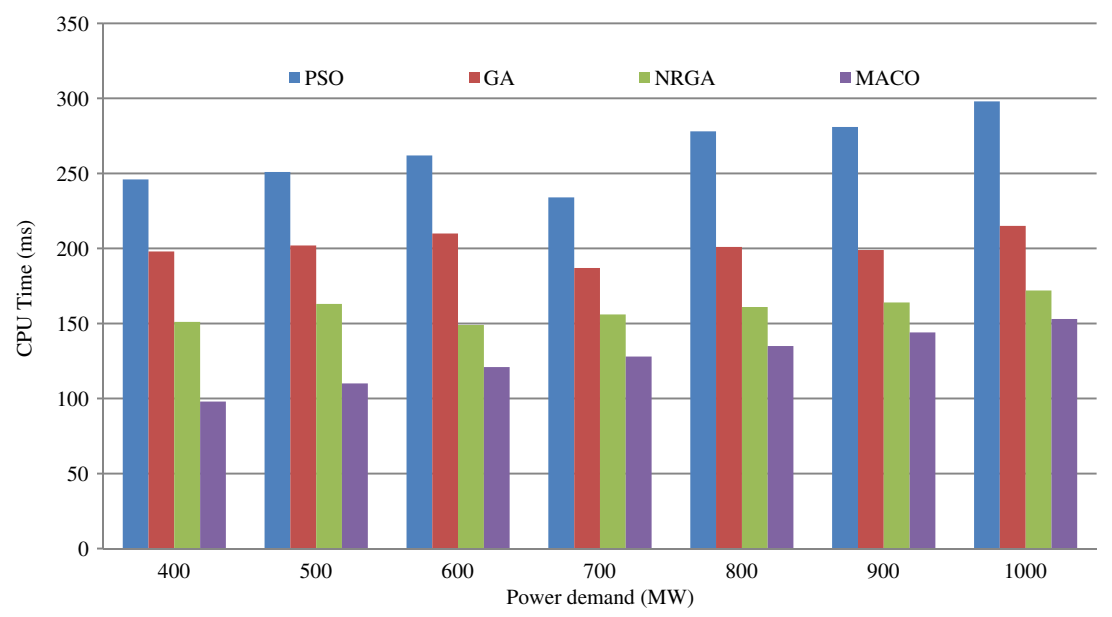

Figure 2. Comparison of the proposed technique by means of required CPU time.

with the results of using optimization techniques such as Particle Swarm Optimization (PSO), Genetic Algorithm (GA) and Non-Dominated Ranked Genetic Algorithm (NRGA). The fuel cost required by various techniques is provided in table 4 . The result clearly suggests that the proposed technique is better as it involved lesser cost for the system operation when compared to other techniques.

The emission resulting using PSO, GA, NRGA and MACO for various power demands is provided in table 5. The emission resulting from using the proposed optimization technique is much reduced when compared to the other techniques. This case is true not only for particular power demand, rather it is true for all cases.

The number of iterations required for optimization using PSO, GA, NRGA and MACO is provided in table 6 and figure 1. It can be observed that the proposed technique required lesser iterations for optimization when compared to other techniques. Also, the time required for optimization is much reduced for the proposed technique when compared to other techniques. It is represented in table 7 and figure 2 .

By considering the overall result, it can be suggested that the usage of proposed technique will reduce the fuel cost as well as the emission output. Also, the time required by the proposed technique is much reduced when compared to the other conventional techniques.

\section{Conclusion}

Optimization algorithms are observed to provide significant results for CEED. By controlling the cost, the pollution causing emissions rises. Therefore, the cost reduction must be controlled by means of a technique called emission dispatch. But, the controlling of emission will increase the cost required for power generation. So, a combined technique called Combined Economic and Emission Dispatch emerges. Various techniques to deal with CEED problem have been suggested by various researchers. The existing system has its own advantages and disadvantages. Widely used technique for CEED problem is to optimize the parameters used for power generation with the help of optimization techniques such as PSO, GA, etc. But, all those techniques 
require more time for optimization. To overcome those difficulties, this study uses MACO optimization technique. The simulation result shows the performance of the proposed technique and it can be suggested that the proposed technique reduces the fuel cost as well as the emission output. Further, it takes lesser time and number of iterations for optimization.

\section{References}

Abido M A 2003 Environmental/economic power dispatch using multiobjective evolutionary algorithms. IEEE Trans. Power Syst. 18(4): 1529-1537

Alrashidi M R and El-Hawary M E 2008 Impact of loading conditions on the emission-economic dispatch. World Academy of Science, Engineering and Technology 29: 148-151

AL-salami Nada M A and Saad Ghaleb Yaseen 2008 Ant colony optimization. IJCSNS Int. J. Comput. Sci. Network Security 8(6): 351-357

Ayoub A K and Patton A D 1971 Optimal thermal generating unit commitment. IEEE Trans. Power Appl. Syst. 90(4): 1752-1756

Chaturvedi K T, Pandit M and Srivastava L 2008 Modified neo-fuzzy neuron-based approach for economic and environmental optimal power dispatch. Appl. Soft. Comp. 8(4): 1428-1438

Chowdhury Badrul H and Rahman S 1990 A review of recent advances in economic dispatch. Institute of Electrical and Electronics Engineers 5(4): 1248-1259

Dhillon J S, Parti S C and Kothari D P 1993 Stochastic economic emission load dispatch. Electric Power Syst. Res. 26: 179-186

Dorigo M, Birattari M and Stitzle T 2006 Ant colony optimization. Arificial Ants as a Computational Intelligence Technique, IEEE Comput. Intell. Mag.

Dos Santos Coelho L, de Andrade Bernert D L, Mariani V C 2010 Chaotic differential harmony search algorithm applied to power economic dispatch of generators with multiple fuel options. IEEE Congress on Evolutionary Computation (CEC), pp. 1-5

Fernando C and Teresa V 2008 Simple ant routing algorithm. Proceedings of International Conference on Information Networking, 486-493, Busan, South Korea

Hui Fan, Zhen Hua, Jin-Jiang Li and Da Yuan 2004 Solving a shortest path problem by ant algorithm. Proceedings of the Third International Conference on Machine Learning and Cybernetics, Shanghai, 26-29 August

King T D, El-Harwary M E and El-Hawary F 1995 Optimal environmental dispatching of electric power systems via an improved hopfield neural network model. IEEE Trans. Power Syst. 10(3): 1559-1565

Lakshmi Devi and Vamsi Krishna O 2008 Combined economic and emission dispatch using evolutionary algorithms-a case study. ARPN J. Eng. Appl. Sci. 3(6): 28-35

Liladhur G Sewothul, Robert T F, Ah King and Harry C S Rughooputh 2004 Genetic algorithms for economic dispatch with valve point effect. Proceedings of the IEEE, International Conference on Networking, Sensing \& Control, pp. 1358-1362

Lowery P G 1966 Generating unit commitment by Dynamic programming. IEEE Trans. Power Appl. Syst. 85(5): 422-466

Peng Chen, Chunhua Zhao, Jian Li and Zhiming Liu 2009 Solving the economic dispatch in power system via a modified genetic particle swarm optimization. International Joint Conference on Computational Sciences and Optimization (CSO) 1: 201-204

Ratniyomchai T, Oonsivilai A, Pao-la-or P and Kulworawanichpong T 2010 Particle swarm optimization for solving combined economic and emission dispatch problems. 5th Proceedings IASME / WSEAS International Conference on Energy \& Environment, pp. 211-216

Sandalidis H, Mavromoustakis K and Stavroulakis P 2004 Ant-based probabilistic routing with pheromone and antipheromone mechanisms. Int. J. Commun. Syst. (IJCS) 17: 55-62

Singh L and Dhillon J S 2008 Secure multiobjective real and reactive power allocation of thermal power units. Int. J. Electric. Power Energ. Syst. 30(10): 594-602 
Song Y H, Morgan R and Williams D 1995 Environmentally constrained electric power dispatch with genetic algorithm. Proc. IEEE IECE, Perth

Song Y H, Wang G S, Wang P Y and Johnst A T 1997 Environmental/economic dispatch using fuzzy logic controlled genetic algorithms. IEEE Proceedings-Generation, Transmission, Distribution 144(4): $377-382$

Talaq J H, El-Hawary F and El-Hawary M E 1994 Minimum emission power flow. IEEE Trans. Power Syst. 9(1): 429-435

Ugur Guvenc 2010 Combined economic emission dispatch solution using genetic algorithm based on similarity crossover. Sci. Res. Essays 5(17): 2451-2456

Wong K P and Yuryevich J 1998 Evolutionary programming-based algorithm for environmentally constrained economic dispatch. IEEE Trans. Power Syst. 13: 301-306 